\title{
Total ischemic time and short, intermediate and long term mortality of patients with STEMI treated by primary percutaneous coronary intervention: Analysis of data from 2004-2013 ACSIS registry
}

Lubovich A, Radzishevsky E, Goldenberg I, Matezky S and Rosenschein U

Department of Cardiology, Center Medical Zion Bnai, Haifa 33394, Israel

\begin{abstract}
Objective: Our objective was to evaluate correlation between mortality and total ischemic time in a large cohort of ST Elevation Myocardial Infarction (STEMI) patients.

Background: Several previous studies demonstrated positive correlation between door to balloon time and mortality.

However, several recent studies failed to find improvement in mortality with shortened door to balloon time. It is possible that further reduction in mortality of STEMI patients in the modern era of PPCI and adjuvant pharmacotherapy could be achieved only by means of reduction of total ischemic time.

Methods: We analyzed data of 2254 consecutive patients with STEMI treated by PPCI and enrolled in the Acute Coronary Syndrome Israeli Survey (ACSIS) registry. We divided our cohort into tertiles based on the total ischemic time: less than 150 minutes (group 1, n=730), between 150 and 265 minutes (group 2, n=758) and above 265 minutes (group 3,n=766). Our primary end points were 30-day, 1-year and 5 years mortality.

Our secondary end point was LVEF less than $40 \%$ at discharge.

Results: There was no difference in 30-day or 1-year mortality between the three study groups (30-day mortality: 3\% vs. 4\% vs. 5\%; 1-year mortality: 6\% vs. 7\% vs. 7\%).

There was significantly lower 5 -year mortality in the shortest (less than 150 minutes) total ischemic time group (11\% vs. $16 \%$ vs. $19 \%)$.

Conclusions: Shortening of total ischemic time below 150 minutes is associated with improved long term survival.

Measures should be undertaken to reduce total ischemic time.
\end{abstract}

\section{Introduction}

During the last decade, emergent performance of primary Percutaneous Coronary Intervention (PCI) became the gold standard treatment for patients with ST elevation myocardial infarction.

The timely performance of the primary PCI as measured by door to balloon time has become one of the main quality measures in the treatment of patients with ST elevation myocardial infarction and is endorsed by both American College of Cardiology and European Society of Cardiology guidelines [1,2].

However, several recent studies, failed to demonstrate improvement in mortality with shortened door to balloon time [3,4].

It is possible that further reduction in mortality of STEMI patients in the modern era of PPCI and adjuvant pharmacotherapy could be achieved only by means of reduction of total ischemic time, which is defined as the time that elapses from chest pain onset until the restoration of coronary blood flow by balloon inflation (or pain to balloon time).
In this study, we sought to examine if there was any correlation between total ischemic time and short, intermediate and long term mortality of patients with STEMI.

\section{Methods}

\section{The ACSIS registry}

Our study was based on the Acute Coronary Syndrome Israeli Survey (ACSIS) registry in which data was prospectively collected from all acute coronary syndrome patients in the State of Israel and operated in collaboration with the Israel Heart Society.

Correspondence to: Lubovich A, Department of Cardiology, Center Medical Zion Bnai, Haifa 33394, Israel; E-mail: alla.lubovich@b-zion.org.il

Key words: percutaneous coronary intervention, myocardial infarction, ischemic time, ACSIS registry

Received: February 02, 2018; Accepted: February 14, 2018; Published: February 17,2018 
Lubovich A (2018) Total ischemic time and short, intermediate and long term mortality of patients with STEMI treated by primary percutaneous coronary intervention: Analysis of data from 2004-2013 ACSIS registry

The ACSIS registry was conducted biennially for a 2-month period during which data on all acute coronary syndrome patients admitted to all coronary care units $(n=25)$ in Israel was provided by each participating center by means of the case report forms (CRF).

The 1-year and 5-years mortality data was adjudicated by a Central Data Coordinating Center through the ministry of internal affairs data set. A Central Data Coordinating Center was responsible for the data collection and analysis.

\section{Study population}

We analyzed data of consecutive patients $(n=2254)$ who presented with STEMI and were treated by PPCI from the 2004,2006,2008,2010 and 2013 ACSIS registries.

We divided our cohort into tertiles based on the total ischemic time: less than 150 minutes (group $1, \mathrm{n}=730$ ), between 150 and 265 minutes (group 2, $\mathrm{n}=758$ ) and above 265 minutes (group 3, $\mathrm{n}=766$ ).

\section{Definitions}

Total Ischemic Time was defined as the time that elapsed from chest pain onset until the restoration of coronary blood flow by balloon inflation (or pain to balloon time).

\section{End points}

Our primary end points were 30-days, 1-year and 5- year's mortality.

Our secondary end point was LVEF less than $40 \%$ at discharge.

\section{Statistical methods}

Direct and adjusted mortality was evaluated with Cox Proportional Hazard model.

The Hazard Ratio (HR) of the upper tertiles versus the lower tertiles is reported along with the $95 \%$ confidence interval.

Adjusted model includes: Age, Sex, Past PCI, Past CVA/TIA and Current Smoking.

Finally the Kaplan Meier crude survival curve was graphically presented along with the $\mathrm{p}$-value from the pairwise log-rank-test. No correction for multiple testing was performed and $\mathrm{p}$-value $<0.05$ was considered statistically significant.

\section{Results}

\section{The basic clinical and demographic characteristics}

The basic clinical and demographic characteristics of the three study groups are presented in Table 1 . There was a significant difference in mean age, gender, frequency of prior PCI, smoking, diabetes mellitus and family history of CAD between the three study groups. There was higher mean age and frequency of diabetes mellitus and lesser frequency of male gender in the group 3 and higher frequency of prior PCI, smoking and family history of CAD in the group 1 .

\section{The short, intermediate and long term mortality}

The 30 day mortality data was available for all patients.

The 1 year mortality data was available for 2233 out of 2254 patients.

The 5 years mortality data was available for 1627 out of 2254 patients because it was not available yet for the 2013 ACSIS cohort.

There was no difference in 30 day and 1 year mortality between the three groups (Table 2).
Table 1. Basic clinical and demographic characteristics of the three study groups

\begin{tabular}{|c|c|c|c|c|}
\hline $\begin{array}{c}\text { Clinical } \\
\text { characteristics }\end{array}$ & $\begin{array}{c}\text { Group 1 } \\
(\mathbf{n = 7 3 0})\end{array}$ & $\begin{array}{c}\text { Group 2 } \\
(\mathbf{n = 7 5 8})\end{array}$ & $\begin{array}{c}\text { Group 3 } \\
(\mathbf{n = 7 6 6})\end{array}$ & $\mathbf{p}$ \\
\hline Mean age & $59.3 \pm 12.4$ & $60.6 \pm 12.3$ & $62.1 \pm 12.4$ & $<.001$ \\
\hline Male & $620(85)$ & $622(82)$ & $611(80)$ & 0.003 \\
\hline Mean weight (kg) & $81.2 \pm 15.7$ & $80.3 \pm 15.5$ & $79.3 \pm 15.0$ & 0.076 \\
\hline Prior MI & $157(22)$ & $148(20)$ & $140(18)$ & 0.274 \\
\hline Prior CABG & $11(2)$ & $21(3)$ & $22(3)$ & 0.018 \\
\hline Prior PCI & $167(23)$ & $156(21)$ & $127(17)$ & 0.009 \\
\hline Family history of & $242(36)$ & $214(29)$ & $191(26)$ & $<.001$ \\
\hline CAD & $453(63)$ & $457(60)$ & $452(59)$ & 0.347 \\
\hline Dyslipidemia & $337(46)$ & $370(49)$ & $402(53)$ & 0.061 \\
\hline Hypertension & $150(21)$ & $214(28)$ & $252(33)$ & $<.001$ \\
\hline Diabetes & $380(53)$ & $384(51)$ & $339(44)$ & 0.004 \\
\hline Current Smoker & $113(16)$ & $135(18)$ & $141(18)$ & 0.330 \\
\hline Past Smoker & & &
\end{tabular}

Table 2. 30 days and 1- year mortality in the three study groups

\begin{tabular}{|c|c|c|c|}
\hline & $\mathbf{3 0}$ days mortality (\%) & 1 year mortality(\%) & $\mathbf{p}$ \\
\hline Total ischemic time $<\mathbf{1 5 0}$ & 3 & 6 & NS \\
\hline $\mathbf{1 5 0} \leq$ Total ischemic time $\leq \mathbf{2 6 5}$ & 4 & 7 & NS \\
\hline Total ischemic time $>\mathbf{2 6 5}$ & 5 & 7 & NS \\
\hline
\end{tabular}

There was significant reduction in 5-years mortality in the group 1 (Table 3).

The shortest (less than 150 minutes) total ischemic time was associated with significantly better 5 years survival (Figure 1).

The cox crude model for 5 years mortality demonstrated that total ischemic time longer than 150 minutes was associated with more than 50 percent increase in 5 years mortality $(\mathrm{HR}=1.51)$ and total ischemic time longer than 265 minutes was associated with more than 80 percent increase in 5 years mortality $(\mathrm{HR}=1.82)$ as compared with total ischemic time of less than 150 minutes (Table 4).

\section{The frequency of LVEF less than $40 \%$ at discharge}

There was significantly less LV dysfunction at discharge in the group 1 than in the groups 2 and 3 ( 32 percent $v s 34$ percent, $\mathrm{p}<0.005$ ).

\section{Discussion}

Since the establishing of the benefit of primary PCI over thrombolytic therapy for the treatment of acute STEMI at the beginning of the 21 century, both ACC/AHA and ESC guidelines endorsed door to balloon time less than 90 minutes being the main performance measure in the treatment of STEMI patients $[1,2]$.

However, several recent studies failed to demonstrate reduction in mortality with shortened door to balloon time.

Lubovich A, et al. compared clinical outcomes and short and intermediate term mortality among STEMI patients admitted directly to CCU or cath lab (the bypass ER group) to STEMI patients brought to ER (the ER group). Despite impressive reduction in door to balloon time (59 vs. 97 minutes), there was no reduction in in MACCE or mortality in the bypass ER group [(4].

Menees DS, et al. analyzed annual trends in door-to-balloon times and in-hospital mortality using data from 96,738 admissions for patients undergoing primary PCI for STEMI from July 2005 through June 2009 at 515 hospitals participating in the CathPCI Registry. Despite improvements in door-to-balloon times from 83 minutes in the 12 months from July 2005 through June 2006 to 67 minutes in the 12 months from July 2008 through June 2009, there was no significant 


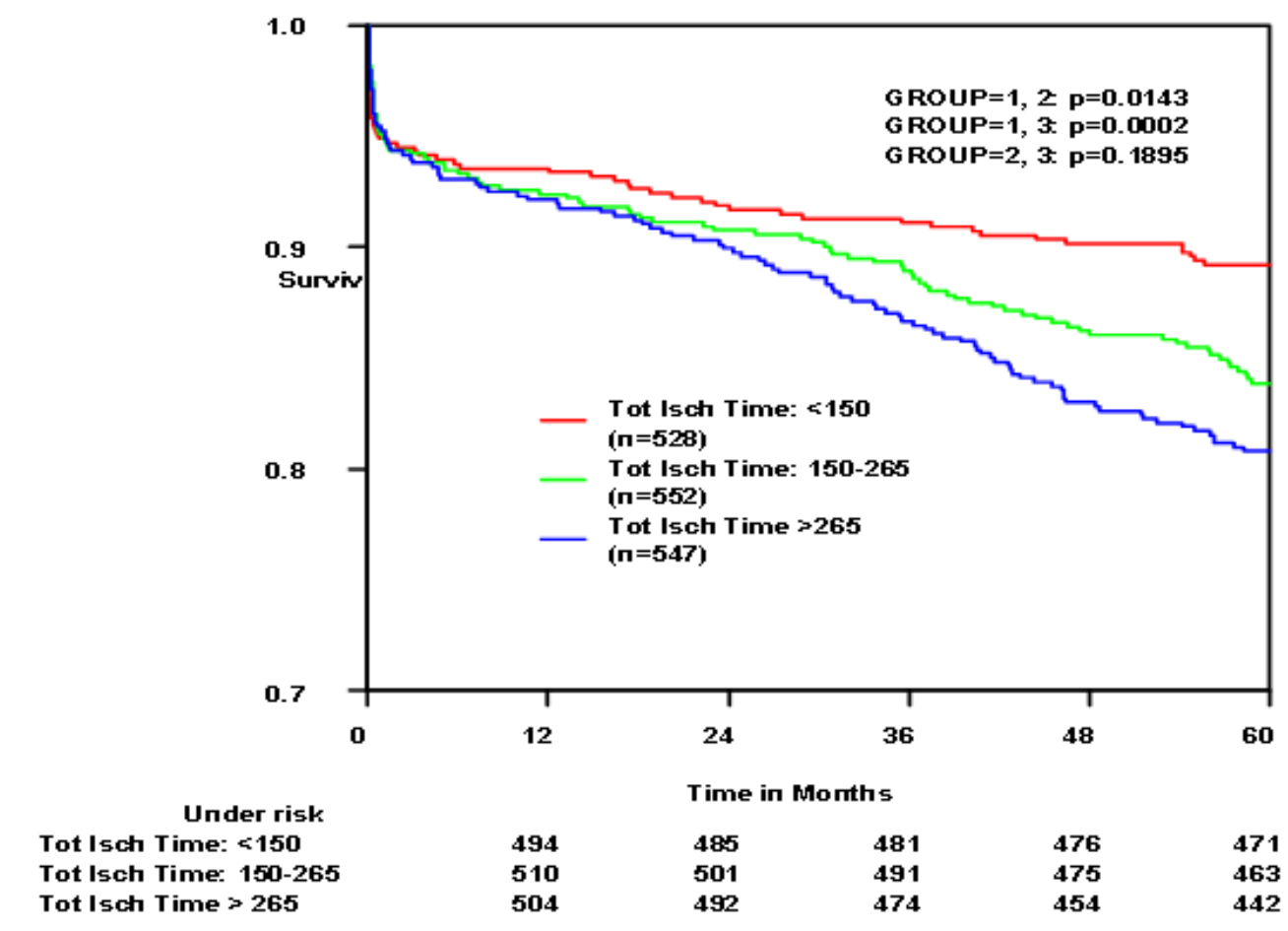

Figure 1. Kaplan Meir Curve for 5 years survival in the three study group.

Table 3. 5 - Years mortality in the three study groups

\begin{tabular}{|c|c|c|}
\hline & 5 -Years' mortality $(\%)$ & $\mathrm{p}$ \\
\hline Total ischemic time $<150$ & 11 & \\
\hline $150 \leq$ Total ischemic time $\leq 265$ & 16 & $\mathrm{P}<0.01$ \\
\hline Total ischemic time $>265$ & 19 & $\mathrm{P}<0.001$ \\
\hline
\end{tabular}

Table 4. Cox crude model for 5 years mortality

\begin{tabular}{|c|c|c|}
\hline & HR & CI\%95 \\
\hline Tot. ischemic time: $150-265$ & 1.51 & $1.08-2.11$ \\
\hline Tot. ischemic time $>266$ & 1.82 & $1.32-2.52$ \\
\hline
\end{tabular}

overall change in unadjusted and risk-adjusted in-hospital mortality and in unadjusted 30-day mortality [3].

The total ischemic time represents the time interval which elapses from chest pain onset till successful opening of the artery and coronary flow restoration by balloon angioplasty.

In this large cohort of STEMI patients, we demonstrated that the shortest, less than 150 minutes, total ischemic time, was associated with reduced long term mortality.

Our findings are supported by the results of several other studies.

G De Luca, et al. analyzed 1 -year mortality of 1,791 patients with STEMI treated by primary angioplasty. They demonstrated that symptom-onset-to-balloon time $>4 \mathrm{~h}$ was an independent predictor of one-year mortality [5].

Solhpour A, et al. reported data of 786 patients with STEMI treated in their center. The patients were divided into three groups according to total ischemic time and four groups according to door to balloon time. They demonstrated that 30-day mortality rate significantly increased across the total ischemic time groups but did not correlate with the door to balloon time groups [6].
Based on our data, we suggest that further national efforts should be concentrated on reduction of total ischemic time rather than on door to balloon time which is only one of its components. The goal should be to get rid of all the unnecessary steps in the care of STEMI patients and develop the systems of care with the focus of decreasing the total ischemic time.

We suggest several measures which could assist in achieving the goal of reduction of the total ischemic time. First of all, we should implement educational measures by using all available social media to decrease the pain onset to EMS call time to less than 30 minutes and to prevent self-referral of patients with chest pain to the ER. Second, we should implement protocols for direct cath lab activation by the field EMS team. Thirdly, we should endorse the first medical contact to balloon time rather than door to balloon time as a national medical services quality measure.

\section{Limitations}

Our study has several important limitations. First of all, it was not a randomized control study. Secondly, the long term mortality data was available only for 72 percent (1627 out of 2254) of the patients. Thirdly, the pain perception is a very subjective matter so the beginning of the pain as perceived by the patient could not represent the exact moment of the coronary artery occlusion.

\section{Conclusion}

Shortening of total ischemic time below 150 minutes is associated with improved long term survival. Measures should be undertaken to reduce total ischemic time. 
Lubovich A (2018) Total ischemic time and short, intermediate and long term mortality of patients with STEMI treated by primary percutaneous coronary intervention: Analysis of data from 2004-2013 ACSIS registry

\section{References}

1. Task Force on the management of ST-segment elevation acute myocardial infarction of the European Society of Cardiology (ESC), Steg PG, James SK, Atar D, Badano LP, et al. (2012) ESC Guidelines for the management of acute myocardial infarction in patients presenting with ST-segment elevation. Eur Heart J 33, 2569-2619. [Crossref]

2. O'Gara PT, Kushner FG, Ascheim DD, Casey DE Jr, Chung MK, et al. (2013) 2013 ACCF/AHA guideline for the management of ST-elevation myocardial infarction: report of the American College of Cardiology Foundation/American Heart Association Task Force on Practice Guidelines. Circulation 127: e362-425. [Crossref]

3. Menees DS, Peterson ED, Wang Y, Curtis JP, Messenger JC, et al. (2013) Door-toBalloon Time and Mortality among Patients Undergoing Primary PCI. $N$ Engl J Med 369: 901-909. [Crossref]
4. Lubovich A1, Dobrecky-Mery I, Radzishevski E, Samnia N, Matetzky S, et al. (2015) Bypassing the emergency room to reduce door-to-balloon time and improve outcomes of ST elevation myocardial infarction patients: analysis of data from 2004-2010 ACSIS registry. J Interv Cardiol 28: 141-146. [Crossref]

5. De Luca G, Suryapranata H, Zijlstra F, van 't Hof AW, Hoorntje JC, et al. (2003) Symptom-onset-to-balloon time and mortality in patients with acute myocardial infarction treated by primary angioplasty. J Am Coll Cardiol 42: 991-997. [Crossref]

6. Solhpour A, Chang KW, Arain SA, Balan P, Loghin C, et al. (2016) Ischemic time is a better predictor than door-to-balloon time for mortality and infarct size in ST-elevation myocardial infarction. Catheter Cardiovasc Interv 87: 1194-1200. [Crossref]

Copyright: $₫ 2018$ Lubovich A. This is an open-access article distributed under the terms of the Creative Commons Attribution License, which permits unrestricted use, distribution, and reproduction in any medium, provided the original author and source are credited. 